\title{
On the Relative Succinctness of Two Extensions by Definitions of Multi-Modal Logic
}

\author{
Wiebe van der Hoek ${ }^{1}$, Petar Iliev ${ }^{1}$, and Barteld Kooi ${ }^{2}$ \\ ${ }^{1}$ University of Liverpool, UK $\{$ wiebe, pvi\}@liverpool.ac.uk \\ ${ }^{2}$ University of Groningen, the Netherlands B.P.Kooi a rug.nl
}

\begin{abstract}
The growing number of logics has lead to the question: How do we compare two formalisms? A natural answer is: We can compare their expressive power and computational properties. There is, however, another way of comparing logics that has attracted attention recently, namely in terms of representational succinctness, i.e. we can ask whether one of the logics allows for a more "economical" encoding of information than the other. Using a suitable version of the so called Formula-Size games, we prove that introducing two simple abbreviations to multi-modal logic leads to an exponential increase in succinctness.

Keywords: epistemic logic, artificial intelligence, multi-agent systems, succinctness, finite model theory of modal logics, formula-size games
\end{abstract}

\section{Introduction}

Consider the following general question. Let $L_{1}$ and $L_{2}$ be two formalisms that express the same class of "properties" or "situations". Is one of the formalisms representationally more succinct (allowing for a more "economical" representation of information) than the other and by how much? A famous instance of this general question is whether there is a family of Boolean functions for which boolean circuits can be exponentially more succinct than boolean formulas. It is widely believed that the answer to this question is beyond our current mathematical knowledge and techniques. Indeed, it is convincingly argued in [5] that we are far from understanding representational succinctness, and we need to first master some very basic problems in order to develop our intuition and mathematical toolbox.

Here we prove that the multimodal logic $[\wedge \Gamma] M L$ is exponentially more succinct than the logic $[\vee \Gamma] M L$ and vice versa. Hence, there are semantic properties that are more economically expressed by using the $[\wedge \Gamma]$ operator; similarly, there are semantic properties that are more economically expressed by using the $[\vee \Gamma]$ operator.

A formula of the form $[\wedge \Gamma] \phi$ is an abbreviation of the multi-modal logic $(M L)$ formula $\bigwedge_{a \in \Gamma}[a] \phi$, where $\Gamma$ is a finite set of relation indices. In the same way, a formula of the form $[\vee \Gamma] \phi$ is just an abbreviation of the $M L$ formula $\bigvee_{a \in \Gamma}[a] \phi$. Readers familiar with epistemic logic will notice that the $[\wedge \Gamma]$ modality corresponds to the "everybody knows" operator, while $[\vee \Gamma]$ corresponds to the "somebody knows" operator. It is obvious that adding formulas of the form $[\wedge \Gamma] \phi$ to $M L$ and, thus, obtaining the logic $[\wedge \Gamma] M L$ does not lead to an increase in expressive power; what is more, the computational complexity of the satisfiability problem for $[\wedge \Gamma] M L$ is the same as that for $M L$ [6]. 
In the same way, $[\vee \Gamma] M L$ is not more expressive than $M L$. However, it is known that both $[\wedge \Gamma] M L$ and $[\vee \Gamma] M L$ are exponentially more succinct than $M L$ [3]. This shows that abbreviations are not just "syntactic sugar" but a genuinely powerful tool. Proving that $[\wedge \Gamma] M L$ is exponentially more succinct than $[\vee \Gamma] M L$ and vice versa, is a further continuation of the line of work started in [3]. Furthermore, it shows that formulas of the form $[\vee \Gamma] \phi$ and $[\wedge \Gamma] \phi$ introduce two different types of "information" compression.

\section{Preliminaries}

\subsection{Multi-modal Logic}

Definition 1 (Multi-modal $[\vee \Gamma][\wedge \Gamma] M L_{n}^{m}$ logic). The signature of the multi-modal logic $[\vee \Gamma][\wedge \Gamma] M L_{n}^{m}$ is a pair $S=\{P, I\}$, where $P=\left\{p_{0}, p_{1} \ldots p_{n}\right\}$ is a finite set of propostional symbols and $I=\left\{a_{1}, \ldots, a_{m}\right\}$ is a finite set of indices. Let $\mathcal{P} I$ be the set of non-empty sub-sets of $I$. The formulas of $[\vee \Gamma][\wedge \Gamma] M L_{n}^{m}$ are built according to the following rules:

$$
\psi:=\perp|\top| p \in P|\neg \psi| \psi \vee \psi|\psi \wedge \psi|[a] \psi|\langle a\rangle \psi|[\vee \Gamma] \psi \mid[\wedge \Gamma] \psi,
$$

where $a \in I$ and $\Gamma \in \mathcal{P} I$.

Definition $2\left([\wedge \Gamma] M L_{n}^{m}\right.$, [ $\left.\vee \Gamma\right] M L_{n}^{m}$, and $\left.M L_{n}^{m}\right)$. The formulas of the logic $[\wedge \Gamma] M L_{n}^{m}$ are the formulas of the logic $[\vee \Gamma][\wedge \Gamma] M L_{n}^{m}$ with the exception of all formulas of the form $[\vee \Gamma] \psi$. Similarly, the formulas of the logic $[\vee \Gamma] M L_{n}^{m}$ are the formulas of the logic $[\vee \Gamma][\wedge \Gamma] M L_{n}^{m}$ with the exception of all formulas of the form $[\wedge \Gamma] \psi$. Finally, the formulas of multi-modal logic $M L_{n}^{m}$ are the formulas of $[\vee \Gamma][\wedge \Gamma] M L_{n}^{m}$ logic with no formulas of the forms $[\wedge \Gamma] \psi$ and $[\vee \Gamma] \psi$ allowed.

The semantics of $[\vee \Gamma][\wedge \Gamma] M L_{n}^{m}$ is given via Kripke models.

Definition 3 (Kripke Model). A Kripke model for the signature $S=\{P, I\}$ is a triple $M=\langle W, R, V\rangle$, where

- W is a set of points;

$-R: I \rightarrow 2^{W \times W}$ is a function from the set of indices $I$ to the power set of $W \times W$, i.e, $R(a)$ is a relation on $W$. We write $w R_{a} v$ for $(w, v) \in R(a)$, and say that $v$ can be reached from $w$ in one a-step.

- $V: P \rightarrow 2^{W}$ is a function from $P$ to $2^{W}$ that assigns a subset $V(p) \subseteq W$ to every $p \in P$.

A model $M=\langle W, R, V\rangle$ is said to be finite if $W$ is finite. Given a model $M=\langle W, R, V\rangle$, a pointed model is a pair $(M, w)$, where $w \in W$. Sets of pointed models are denoted $\mathbb{M}$, $\mathbb{N}, \mathbb{M}_{1}, \mathbb{N}_{1}, \mathbb{M}_{2}, \mathbb{N}_{2}$, etc.

We define the notion "formula $\phi$ is true in a pointed model $(M, w)$ " in the usual way (see for example [2]). In particular:

$$
\begin{aligned}
& (M, w)=\langle a\rangle \psi \quad \text { iff there is a } v \in W \text { such that } w R_{a} v \text { and }(M, v) \models \psi ; \\
& (M, w)=[a] \psi \quad \text { iff }(M, v) \models \psi \text { for all } v \in W \text { such that } s R_{a} v ; \\
& (M, w)=[\wedge \Gamma] \psi \text { iff }(M, w)=\bigwedge_{a \in \Gamma}[a] \psi ; \\
& (M, w) \mid=[\vee \Gamma] \psi \text { iff }(M, w) \models \bigvee_{a \in \Gamma}[a] \psi
\end{aligned}
$$


Given the semantics of the operators " $[\vee \Gamma]$ " and " $[\vee \Gamma]$ ", it is obvious that $[\wedge \Gamma] M L_{n}^{m}$, $[\vee \Gamma] M L_{n}^{m}$, and $[\vee \Gamma][\wedge \Gamma] M L_{n}^{m}$ are just extensions by definition of the logic $M L_{n}^{m}$. In addition, if $\Gamma=\{a\}$, then both $[\wedge \Gamma] \psi$ and $[\vee \Gamma] \psi$ are just the formula $[a] \psi$. Hence, we assume that the set $\Gamma$ contains at least two indices.

As usual, for every pointed model $(M, w)$, and any formula $\langle a\rangle \psi$,

$$
(M, w) \models\langle a\rangle \psi \leftrightarrow \neg[a] \neg \psi .
$$

From now on, if $(M, w)$ is a pointed model, where $M=\langle W, R, V\rangle$, we write $v \in M$ instead of $v \in W$; all models and all sets of pointed models are finite. We define the following operations on pointed models and sets of pointed models:

Definition 4. Let $(M, w)$ and $\mathbb{M}=\left\{\left(M_{1}, w_{1}\right), \ldots,\left(M_{k}, w_{k}\right)\right\}$ be a pointed model and a set of pointed models for the signature $S=\{P, I\}$. Let $\Gamma=\left\{a_{i}, \ldots, a_{j}\right\}$ be a subset of $I$. Then

- $[a](M, w)=\left\{(M, v) \mid v \in M\right.$ and $\left.w R_{a} v\right\}$. Intuitively, $[a](M, w)$ is the set of all pointed models that can be reached from $w$ by making one a-step. Note that if there is no point $v \in M$ such that $w R_{a} v$, then $[a](M, w)=\emptyset$.

- $[a] \mathbb{M}=[a]\left(M_{1}, w_{1}\right) \cup \ldots \cup[a]\left(M_{k}, w_{k}\right)$.

- $[\wedge \Gamma] \mathbb{M}=\left[a_{i}\right] \mathbb{M} \cup \ldots \cup\left[a_{j}\right] \mathbb{M}$, i.e., $[\wedge \Gamma] \mathbb{M}$ is the union of $[a] \mathbb{M}$ for all $a \in \Gamma$.

Given a class of pointed models $\mathbb{C}$, we write $\mathbb{C} \models \varphi$ to mean that for all $(M, w) \in$ $\mathbb{C},(M, w) \models \varphi$. Note that if $\mathbb{C}=\emptyset$, then for every $[\vee \Gamma][\wedge \Gamma] M L_{n}^{m}$ formula $\phi$, it is trivially true that $\mathbb{C}=\phi$. In Section 3 we are going to use the well known fact that if two pointed models $(M, w)$ and $(N, v)$ are bisimilar, then for every $[\vee \Gamma][\wedge \Gamma] M L_{n}^{m}$ formula $\phi,(M, w) \models \phi$ if and only if $(N, v) \models \phi$ (see [2]).

Definition 5. Let $\mathbb{C}$ be a non-empty class of models. We say that logic $L_{1}$ is at least as expressive as logic $L_{2}$ on the class of models $\mathbb{C}$, written $L_{2} \leq_{\mathbb{C}}^{\text {exp }} L_{1}$, if for every $\varphi_{2} \in L_{2}$, there is a formula $\varphi$ in $L_{1}$ such that $\mathbb{C}=\varphi_{1} \leftrightarrow \varphi_{2}$.

\subsection{Extended Syntax Trees}

Every $[\vee \Gamma][\wedge \Gamma] M L_{n}^{m}$ formula $\phi$ can be represented by a syntax tree in the usual way, i.e., every leaf of the tree is labeled with an atomic symbol occurring in $\phi$ and inner nodes are labeled with Boolean connectives or modal operators occurring in $\phi$.

The notion of extended syntax tree of a first-order logic formula was introduced in [5]. Extended syntax trees correspond to game trees for the Adler-Immerman games defined in [1]. The reader can think about these trees as normal syntax trees in which every node has an additional semantic label that consists of two sets of pointed models.

Definition 6 (Extended Syntax Tree). Let $\phi$ be a $[\vee \Gamma][\wedge \Gamma] M L_{n}^{m}$ formula and let $\mathbb{M}$ and $\mathbb{N}$ be two sets of pointed models such that $\mathbb{M} \models \phi$ and $\mathbb{N} \models \neg \phi$. An extended syntax tree $T_{\phi}^{\langle\mathrm{M}, \mathbb{N}\rangle}$ is defined inductively on the structure of $\phi$ as follows:

( $\phi$ is a propositional symbol $p \in P$ ): $T_{p}^{\langle\mathbb{M}, \mathbb{N}\rangle}$ consists of a single node that has a $\operatorname{syntax}$ label $\operatorname{synl}(t):=p$ and a semantic label $\operatorname{seml}(t):=\langle\mathbb{M}, \mathbb{N}\rangle$. 
( $\phi$ is $\top): T_{\top}^{\langle\mathrm{M}, \mathbb{N}\rangle}$ consists of a single node $t$ with $\operatorname{synl}(t):=\top$ and $\operatorname{seml}(t):=\langle\mathbb{M}, \mathbb{N}\rangle$. Note that in this case $\mathbb{N}=\emptyset$.

$(\phi$ is $\perp): T_{\perp}^{\langle\mathbb{M}, \mathbb{N}\rangle}$ consists of a single node $t$ with $\operatorname{synl}(t):=\perp$ and $\operatorname{seml}(t):=\langle\mathbb{M}, \mathbb{N}\rangle$. Note that in this case $\mathbb{M}=\emptyset$.

$(\phi$ is $\neg \psi): T_{\neg \psi}^{\langle\mathbb{M}, \mathbb{N}\rangle}$ has a root node $t$ with $\operatorname{synl}(t):=\neg$ and $\operatorname{seml}(t):=\langle\mathbb{M}, \mathbb{N}\rangle$. The unique child of $t$ is the root of $T_{\psi}^{\langle\mathbb{N}, \mathbb{M}\rangle}$. Note that $\mathbb{N}=\psi$ and $\mathbb{M}=\neg \psi$.

$\left(\phi\right.$ is $\left.\psi_{1} \wedge \psi_{2}\right): T_{\psi_{1} \wedge \psi_{2}}^{\langle\mathbb{M}, \mathbb{N}\rangle}$ has a root node $t$ with $\operatorname{synl}(t):=\wedge$ and $\operatorname{seml}(t):=\langle\mathbb{M}, \mathbb{N}\rangle$. The first child of $t$ is the root of $T_{\psi_{1}}^{\left\langle\mathbb{M}, \mathbb{N}_{1}\right\rangle}$. The second child of t is the root of $T_{\psi_{2}}^{\left\langle\mathrm{M}, \mathbb{N}_{2}\right\rangle}$, where, for $i \in\{1,2\}, \mathbb{N}_{i}=\left\{(N, v) \mid(N, v) \models \neg \psi_{i}\right\}$.

Note that $\mathbb{N}=\mathbb{N}_{1} \cup \mathbb{N}_{2}, \mathbb{M}=\psi_{1} \wedge \psi_{2}$, and $\mathbb{N}_{1} \models \neg \psi_{1}, \mathbb{N}_{2} \models \neg \psi_{2}$.

$\left(\phi\right.$ is $\left.\psi_{1} \vee \psi_{2}\right): T_{\psi_{1} \vee \psi_{2}}^{\langle\mathbb{M}, \mathbb{N}\rangle}$ has a root node $t$ with $\operatorname{synl}(t):=\vee$ and $\operatorname{seml}(t):=\langle\mathbb{M}, \mathbb{N}\rangle$.

The first child of t is the root of $T_{\psi_{1}}^{\left\langle\mathrm{M}_{1}, \mathbb{N}\right\rangle}$. The second child of $t$ is the root of $T_{\psi_{2}}^{\left\langle\mathrm{M}_{2}, \mathbb{N}\right\rangle}$, where, for $i \in\{1,2\}, \mathbb{M}_{i}=\left\{(M, v)|(M, v)|=\psi_{i}\right\}$.

Note that $\mathbb{M}=\mathbb{M}_{1} \cup \mathbb{M}_{2}, \mathbb{M}_{1}=\psi_{1}$, and $\mathbb{M}_{2}=\psi_{2}, \mathbb{N}=\neg\left(\psi_{1} \vee \psi_{2}\right)$.

$(\phi$ is $[a] \psi): T_{[a] \psi}^{\langle\mathbb{M}, \mathbb{N}\rangle}$ has a root node $t$ with $\operatorname{synl}(t):=[a]$ and $\operatorname{seml}(t):=\langle\mathbb{M}, \mathbb{N}\rangle$.

Since $\mathbb{N} \mapsto\langle a\rangle \neg \psi$, for every $(N, w) \in \mathbb{N}$, there is a $v \in N$ such that $w R_{a} v$ and $(N, v) \models \neg \psi$. Let $\langle a\rangle \mathbb{N}$ be the set of these models. The unique child of $t$ is the root

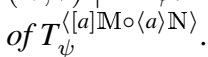

Note that $[a] \mathbb{M}=\psi$ and $\langle a\rangle \mathbb{N}=\neg \psi$.

$(\phi$ is $\langle a\rangle \psi): T_{\langle a\rangle \psi}^{\langle\mathbb{M}, \mathbb{N}\rangle}$ has a root node $t$ with $\operatorname{synl}(t):=\langle a\rangle$ and $\operatorname{seml}(t):=\langle\mathbb{M}, \mathbb{N}\rangle$.

Since $\mathbb{M} \models\langle a\rangle \psi$, for every $(M, w) \in \mathbb{M}$, there is $a v \in M$ such that $w R_{a} v$ and $(M, v) \models \psi$. Let $\langle a\rangle \mathbb{M}$ be the set of these models. The unique child of $t$ is the root of $T_{\psi}^{\langle\langle a\rangle \mathrm{M} \circ[a] \mathrm{N}\rangle}$.

Note that $\langle a\rangle \mathbb{M}=\psi$ and $[a] \mathbb{N}=\neg \psi$.

$(\phi$ is $[\wedge \Gamma] \psi): T_{[\wedge \Gamma] \psi}^{\langle\mathbb{M}, \mathbb{N}\rangle}$ has a root node $t$ with $\operatorname{synl}(t):=[\wedge \Gamma]$ and $\operatorname{seml}(t):=\langle\mathbb{M}, \mathbb{N}\rangle$.

Since $\mathbb{N} \models \neg[\wedge \Gamma] \psi$ it follows that for every $(N, w) \in \mathbb{N}$, there is an $a \in \Gamma$ and $a v \in N$ such that $w R_{a} v$ and $(N, v) \models \neg \psi$. Let $\langle\wedge \Gamma\rangle \mathbb{N}$ be the set of these pointed models.

The unique child of $t$ is the root of $T_{\psi}^{\langle[\wedge \Gamma] \mathrm{M},\langle\wedge \Gamma\rangle \mathrm{N}\rangle}$.

Note that $[\wedge \Gamma] \mathbb{M}=\psi$ and $\langle\wedge \Gamma\rangle \mathbb{N} \models \neg \psi$.

$(\phi$ is $[\vee \Gamma] \psi): T_{[\vee \Gamma] \psi}^{\langle\mathbb{M}, \mathbb{N}\rangle}$ has a root node $t$ with $\operatorname{synl}(t):=[\vee \Gamma]$ and $\operatorname{seml}(t):=\langle\mathbb{M}, \mathbb{N}\rangle$.

Since $\mathbb{N}=\neg[\vee \Gamma] \psi$ it follows that for every $(N, w) \in \mathbb{N}$, and every a $\in \Gamma$, there is $a v \in N$, such that $w R_{a} v$ and $(N, v) \models \neg \psi$. Let $\langle\vee \Gamma\rangle \mathbb{N}$ be the set of these pointed models. On the other hand, $\mathbb{M} \models[\vee \Gamma] \psi$ implies that for every $(M, w) \in \mathbb{M}$, there is an $a \in \Gamma$ such that $[a](M, w) \models \psi$. Let $[\vee \Gamma] \mathbb{M}$ be the set of these models.

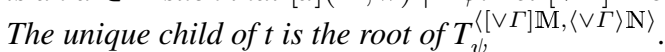

Note that $[\vee \Gamma] \mathbb{M}=\psi$ and $\langle\vee \Gamma\rangle \mathbb{N} \mid=\neg \psi$.

Figure 1 shows the extended syntax tree $T_{q \wedge\langle a\rangle \top}^{\langle\mathbb{M}, \mathbb{N}\rangle}$ of the formula $q \wedge\langle a\rangle \top$, where $\mathbb{M}=\left\{\left(M_{1}, s_{1}\right),\left(M_{2}, s_{2}\right)\right\}$ and $\mathbb{N}=\left\{\left(M_{3}, s_{3}\right),\left(M_{4}, s_{4}\right)\right\}$. Only the atoms true at a given point are shown. Black circles designate the leaves of the tree. The current points in the models are also black.

Then next proposition follows immediately from Definition 6. 
Proposition 1. For any $[\vee \Gamma][\wedge \Gamma] M L_{n}^{m}$ formula $\phi$ and any sets of pointed models $\langle\mathbb{M}, \mathbb{N}\rangle$ such that $\mathbb{M}=\phi$ and $\mathbb{N} \models \neg \phi$, if the root of the extended syntax tree $T_{\phi}^{\langle\mathbb{M}, \mathbb{N}\rangle}$ has a child-node that is the root of the syntax tree $T_{\psi}^{\langle\mathbb{D}, \mathbb{P}\rangle}$, then $\mathbb{D} \models \psi$ and $\mathbb{P}=\neg \psi$.

Definition 7 (Branches). A branch B in an extended-syntax tree is any path leading from the root of the tree to a leaf. We denote by $I(B)$ the word $i_{1} \ldots i_{k}$, formed by the indices of all the nodes with syntactic labels of the form $[i]$ or $\langle i\rangle$ occurring along a branch $B$ when traversing the branch from the root to its leaf.

For instance, the branch $B$ (right) of the game tree of Figure 1 satisfy $I(B)=a b$. Note that given an extended syntax tree $T_{\phi}^{\langle\mathrm{M}, \mathbb{N}\rangle}$, the number of nodes and the geometry of the tree depend solely on $\phi$, e.g., if we disregard the semantic labels of the nodes, the three extended syntax trees $T_{q \wedge\langle a\rangle\langle b\rangle \top \wedge[\vee}^{\langle\mathbb{M}, \mathbb{N}\rangle}, T_{q \wedge\langle a\rangle\langle b\rangle \top \wedge[\vee \Gamma \Gamma] p}^{\langle\emptyset, \mathbb{N}\rangle}$, and $T_{q \wedge\langle a\rangle\langle b\rangle \top \wedge[\vee \Gamma] p}^{\langle\emptyset, \emptyset\rangle}$ are actually the usual syntax tree associated with the formula $q \wedge\langle a\rangle\langle b\rangle \top \wedge[\vee \Gamma] p$, where $\Gamma=\{a, b\}$. Hence, the following definition is unambiguous.

Definition 8 (Formula Size). Let $\phi$ be $a[\vee \Gamma][\wedge \Gamma] M L_{n}^{m}$ formula and let $\mathbb{M}$ and $\mathbb{N}$ be two arbitrary sets of pointed models such that $\mathbb{M} \models \phi$ and $\mathbb{N} \models \neg \phi$. The size of $\phi$ (denoted $\|\phi\|)$ is the number of verticies occurring in $T_{\phi}^{\langle\mathrm{M}, \mathrm{N}\rangle}$.

We enumerate the nodes of an extended syntax tree $T_{\phi}^{\langle\mathbb{M}, \mathbb{N}\rangle}$ in increasing order starting from the root of the tree which is the first node in this enumeration; it's children are enumerated from left to right, etc, i.e., the nodes are enumerated as in a breadth-first search algorithm.

\subsection{Succinctness}

Following [5], our formal definition of the term succinctness is:

Definition 9 (Succinctness). Let $L_{1}, L_{2}$ be two logics. Let $\mathbb{C}$ be a class of models such that $L_{1} \leq_{\mathbb{C}}^{\operatorname{expr}} L_{2}$. Let $\mathcal{F}$ be a class of functions $f: \mathbb{N} \rightarrow \mathbb{R}$. We say that $L_{1}$ is $\mathcal{F}$ succinct in $L_{2}$ on $\mathbb{C}$, and write $L_{1} \leq_{\mathbb{C}}^{\mathcal{F}} L_{2}$, iff there is a function $f \in \mathcal{F}$ such that for every $L_{1}$-formula $\phi_{1}$, there is an $L_{2}$-formula $\phi_{2}$ for which the following is true:

- $\mathbb{C} \models \phi_{1} \leftrightarrow \phi_{2}$

- $\left\|\phi_{2}\right\| \leq f\left(\left\|\phi_{1}\right\|\right)$.

Intuitively, this means that $\mathcal{F}$ gives an upper bound on the size of $L_{2}$ formulas needed to express all of $L_{1}$ on $\mathbb{C}$. Therefore, by saying that $L_{1}$ is exponentially more succinct than $L_{2}$, we mean $L_{1} \leq_{\mathbb{C}}^{\operatorname{expr}} L_{2}$ and $L_{1} \underset{\mathbb{C}}{\Varangle_{\mathbb{C}}^{S U B E X P}} L_{2}$, i.e., the size of formulas of $L_{2}$ expressing all of $L_{1}$ on $\mathbb{C}$ cannot be bounded from above by a sub-exponential function.

In order to prove that $L_{1} \underset{\mathbb{C}}{S U B E X P} L_{2}$ for two logics $L_{1}$ and $L_{2}$, it is sufficient to show that there are two infinite sequences of formulas $\phi_{1}, \phi_{2}, \ldots$ in $L_{1}$ and $\chi_{1}, \chi_{2}, \ldots$ in $L_{2}$, and rational numbers $k$ and $t$ such that

1. $\left\|\phi_{n}\right\|=k n+t$;

2. $\left\|\chi_{n}\right\| \geq 2^{i}$

3. $\chi_{n}$ is the shortest formula in $\mathrm{L}_{2}$ such that $\mathbb{C}=\phi_{n} \leftrightarrow \chi_{n}$. 


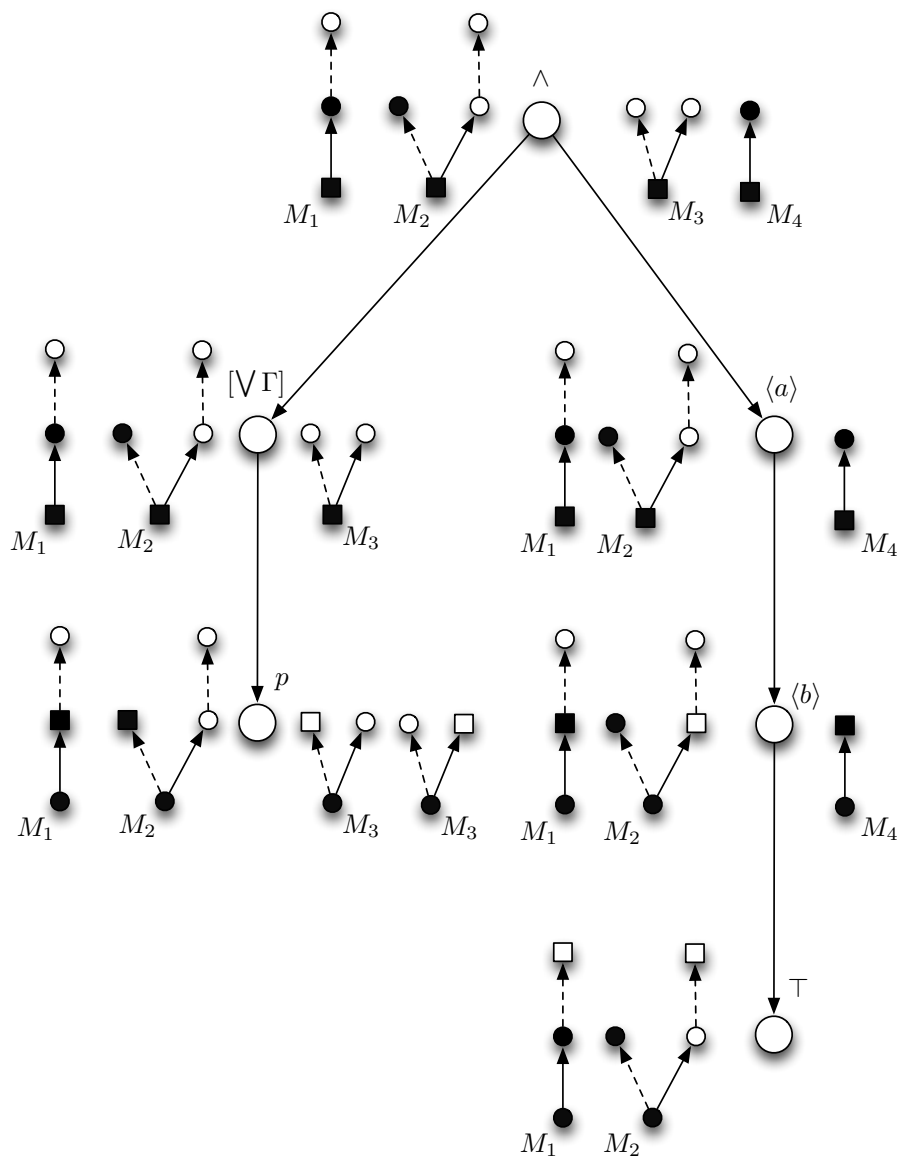

Fig. 1. The extended syntax tree $T_{[\vee \Gamma] p \wedge\langle a\rangle\langle b\rangle \top}^{\langle\mathbb{M}, \mathbb{N}\rangle}$

with $\Gamma=\{a, b\}$. The current point in the pointed Kripke model is denoted by a square, other states are denoted by circles. Black squares and circles denote the only states where atom $p$ is true. The solid arrows between pointed states denote steps for $a$, the dashed arrows belong to $b$. The bigger white circles denote nodes in the syntax tree.

\section{Main Results}

For a natural number $n \geq 1$, let $[\wedge \Gamma]^{n}$ stand for $\overbrace{[\wedge \Gamma] \ldots[\wedge \Gamma]}^{n \text { times }[\wedge \Gamma]}$ and similarly for $[\vee \Gamma]^{n}$.

Definition 10. Let $S=\{P, I\}$ be a signature where $P$ contains at least one propositional symbol $p$ and $I$ contains at least two indices $a$ and $b$ and let $\Gamma=\{a, b\}$. For 
every $n \geq 1$, the $[\wedge \Gamma] M L_{n}^{m}$ formulas $\phi_{n}$, the $[\vee \Gamma] M L_{n}^{m}$ formulas $\theta_{n}$, and the $M L_{n}^{m}$ formulas $\psi_{n}$ and $\chi_{n}$ are defined as follows.

$$
\begin{aligned}
\phi_{n}:=\neg[\wedge \Gamma]^{n} \neg p, & \psi_{1}:=\langle a\rangle p \vee\langle b\rangle p ; \\
\theta_{n}:=[\vee \Gamma]^{n} p, & \psi_{n}:=\langle a\rangle \psi_{n-1} \vee\langle b\rangle \psi_{n-1}, n>1 . \\
& \chi_{1}:=[a] p \vee[b] p ; \\
& \chi_{n}:=[a] \chi_{n-1} \vee[b] \chi_{n-1}, n>1 .
\end{aligned}
$$

Given the fact that $[\wedge \Gamma] p$ and $[\vee \Gamma] p$ are abbreviations of $[a] p \wedge[b] p$ and $[a] p \vee[b] p$ respectively, it is easily seen that the formulas in the right column are equivalent to the formulas in the left column. It is obvious that the length of the latter is linear in $n$ while the length of the former is exponential in $n$.

Firstly, we would like to prove that there is no sequence of $[\vee \Gamma] M L_{n}^{m}$ formulas $\delta_{n}$ such that $\phi_{n}$ is equivalent to $\delta_{n}$ and at the same time the length of $\delta_{n}$ is subexponential in $n$. To this end, for every $n \geq 1$, we define a set of models $\mathbb{M}^{n}$ such that $\mathbb{M}^{n} \models \phi_{n}$. Then we find a set of models $\mathbb{N}^{n}$ such that $\mathbb{N}^{n}=\neg \phi_{n}$. Finally, we prove that for every $[\vee \Gamma] M L_{n}^{m}$ formula $\delta$ such that $\mathbb{M}^{n} \cup \mathbb{N}^{n} \models \phi_{n} \leftrightarrow \delta$, the extended syntax tree $T_{\delta}^{\left\langle\mathbb{M}^{n}, \mathbb{N}^{n}\right\rangle}$ has at least $2^{n}$ nodes. Intuitively, it is clear that the main difficulty in such a proof stems from the "power" of the $[\mathrm{V} \Gamma]$ operator. While $(M, w)=[a] \phi$ means that all points reachable from $w$ in one $a$-step satisfy the formula $\phi,(M, w) \models[\vee \Gamma] \phi$ means that there is an index $i \in \Gamma$ such that all points reachable from $w$ in one $i$-step satisfy $\phi$. Therefore, if we manage to define the models in $\mathbb{M}^{n}$ and $\mathbb{N}^{n}$ in such a way as to make the $[\vee \Gamma]$ operator "useless" our task will be easier.

Secondly, we would like to prove that there is no $[\wedge \Gamma] M L_{n}^{m}$ formula $\gamma_{n}$ of subexponential length such that $\theta_{n}$ is equivalent to $\gamma_{n}$. Again, the main problem is the power of the $[\wedge \Gamma]$ modality. Guided by the same intuition, for every $n \geq 1$, we define sets of models $\mathbb{D}^{n}$ and $\mathbb{P}^{n}$ where $\mathbb{D}^{n}=\theta_{n}, \mathbb{P}^{n}=\neg \theta_{n}$, and for every $[\wedge \Gamma] M L_{n}^{m}$ formula $\gamma$ such that $\mathbb{D}^{n} \cup \mathbb{P}^{n} \models \phi_{n} \leftrightarrow \delta$, the syntax tree $T_{\delta}^{\left\langle\mathbb{D}^{n}, \mathbb{P}^{n}\right\rangle}$ has at least $2^{n}$ nodes. As for $[\vee \Gamma] M L_{n}^{m}$, we define the models in $\mathbb{O}^{n}$ and $\mathbb{P}^{n}$ so that the operator $[\wedge \Gamma]$ is of no "use".

Definition 11 and items (b) and (c) from Lemma 1 are the formalization of this idea.

Definition 11 (Tree models). Let the signature $S=\{P, I\}$ be as in Definition 10. Figure 2 shows the sets of models $\mathbb{M}^{n}, \mathbb{N}^{n}, \mathbb{D}^{n}$, and $\mathbb{P}^{n}$. The tree-like models in $\mathbb{M}^{1}$, $\mathbb{N}^{1}, \mathbb{D}^{1}, \mathbb{P}^{1}$ are built as shown. The models in $\mathbb{D}^{n+1}$ and $\mathbb{P}^{n+1}$, for example, are defined recursively by taking a model from $\mathbb{O}^{1}$, erasing the propositional symbols, and then using the leaves of the tree as roots for the models from $\mathbb{D}^{n}$ and $\mathbb{P}^{n}$ as shown ${ }^{3}$. The same strategy is employed in the construction of the models in $\mathbb{M}^{n+1}$ and $\mathbb{N}^{n+1}$.

We denote the root of any of the tree-like models in $\mathbb{M}^{n}, \mathbb{N}^{n}, \mathbb{O}^{n}, \mathbb{P}^{n}$ by $r$. For any tree-like model $M_{x k}^{n+1}$, such that $x \in\{a, b\}$, the pair $\left(M_{x k}^{n+1}, M_{k}^{n}\right)$ stands for the pointed model $\left(M_{x k}^{n+1}, r\right)$, where $r$ is the root of the model $M_{k}^{n}$. Similarly for $\left(M_{x k}^{n+1}, N^{n}\right)$ and $\left(N^{n+1}, N^{n}\right)$.

\footnotetext{
${ }^{3}$ Intuitively, the subscript in the name of the model $O_{a k}^{n+1}$ encodes a path (starting with an $a$ step) leading from the root of the tree to a leaf satisfying the proposition $p$. The same path in the model $P_{a k}^{n+1}$ leads to a point that does not satisfy $p$. Appart from this difference, the models $O_{a k}^{n+1}$ and $P_{a k}^{n+1}$ look the same.
} 
$\mathbb{M}^{1}$

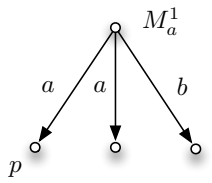

$\mathbb{M}^{n+1}$

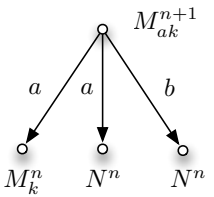

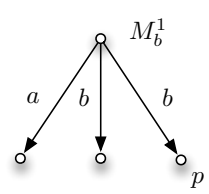

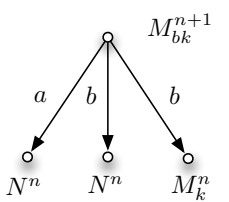

$\mathbb{N}^{1}$

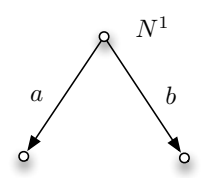

$\mathbb{N}^{n+1}$

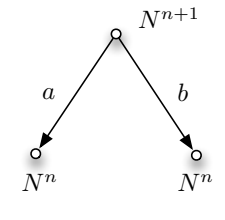

$\mathbb{D}^{1}$

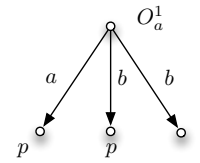

$\mathbb{D}^{n+1}$

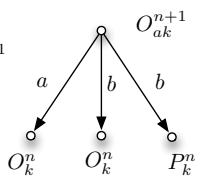

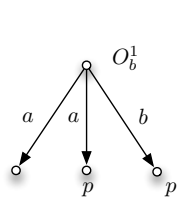
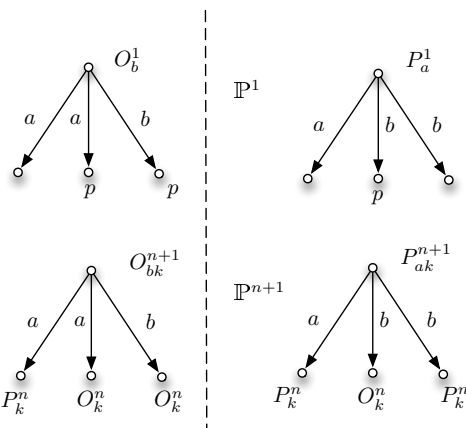

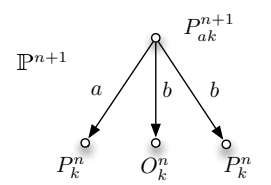

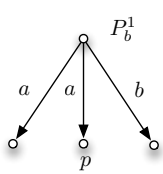

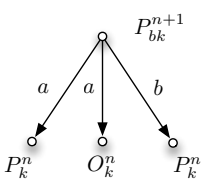

Fig. 2. The sets of models $\mathbb{M}^{n}, \mathbb{N}^{n}, \mathbb{D}^{n}$, and $\mathbb{P}^{n}$.

Lemma 1. Let the sequences of formulas $\psi_{n}$ and $\chi_{n}$ be defined as in Definition 10 and let $\mathbb{M}^{n}, \mathbb{N}^{n}, \mathbb{D}^{n}$, and $\mathbb{P}^{n}$ be as in Definition 11. For every $n$, and every sets of pointed models $\mathbb{S}$ and $\mathbb{T}$ :

(a) $\mathbb{M}^{n} \models \phi_{n}, \mathbb{N}^{n}=\neg \phi_{n}, \mathbb{D}^{n}=\theta_{n}, \mathbb{P}^{n} \models \neg \theta_{n}$;

(b) For any pointed model $\left(M_{w}^{n}, r\right)$, there is neither a formula $[\vee \Gamma] \psi$ such that $\left(M_{w}^{n}, r\right) \models[\vee \Gamma] \psi$ and $\left(N^{n}, r\right) \models \neg[\vee \Gamma] \psi$ nor $[\vee \Gamma] \psi$ such that $\left(N^{n}, r\right) \models[\vee \Gamma] \psi$ and $\left(M_{w}^{n}, r\right) \models \neg[\vee \Gamma] \psi$.

(c) For any pointed model $\left(O_{w}^{n}, r\right)$, there is neither a formula $[\wedge \Gamma] \psi$ such that $\left(O_{w}^{n}, r\right) \models[\wedge \Gamma] \psi$ and $\left(P_{w}^{n}, r\right) \models \neg[\vee \Gamma] \psi$ nor $[\vee \Gamma] \psi$ such that $\left(P_{w}^{n}, r\right) \models[\wedge \Gamma] \psi$ and $\left(O_{w}^{n}, r\right)=\neg[\vee \Gamma] \psi$.

(d) For any $[\vee \Gamma] M L_{n}^{m}$ formula $\phi$ such that $\mathbb{S} \cup\left\{\left(M_{w}^{n}, r\right)\right\} \models \phi$ and $\mathbb{T} \cup\left\{\left(N^{n}, r\right)\right\} \models \neg \phi$, the extended syntax tree $T_{\phi}^{\left\langle\mathbb{S} \cup\left\{\left(M_{w}^{n}, r\right)\right\},\left\{\left(N^{n}, r\right)\right\} \cup \mathbb{T}\right\rangle}$ has a branch $B$, where $I(B)=w$.

(e) For any $[\wedge \Gamma] M L_{n}^{m}$ formula $\phi$ such that $\mathbb{S} \cup\left\{\left(O_{w}^{n}, r\right)\right\} \models \phi$ and $\left\{\left(P_{w}^{n}, r\right)\right\} \cup \mathbb{T} \models \neg \phi$, the extended syntax tree $T_{\phi}^{\left\langle\mathbb{S} \cup\left\{\left(O_{w}^{n}, r\right)\right\},\left\{\left(P_{w}^{n}, r\right)\right\} \cup \mathbb{T}\right\rangle}$ has a branch $B$, where $I(B)=w$.

Proof. 
(a) It is easily seen that $\mathbb{M}^{n} \models \psi_{n}, \mathbb{N}^{n} \models \neg \psi_{n}, \mathbb{D}^{n} \models \chi_{n}, \mathbb{P}^{n} \models \neg \chi_{n}$.

(b) We prove only the case $n>1$ and $w=a k$. The case $w=b k$ or $n=1$ is analogous. Assume there is a formula $[\mathrm{V} \Gamma] \psi$ such that $\left(M_{a k}^{n}, r\right) \models[\vee \Gamma] \psi$ and $\left(N^{n}, r\right)=\neg[\vee \Gamma] \psi$. Consider the extended syntax tree $T_{[\vee \Gamma \mid \psi}^{\left\langle\left\{\left(M_{a k}^{k}, r\right)\right\} \circ\left\{\left(N^{n}, r\right)\right\}\right\rangle}$. Using the last item from Definition 6, we see that there must be two points $s$ and $t$ in $N^{n}$ such that $r R_{a} s$ and $\left(N^{n}, s\right) \models \neg \psi$ and $r R_{b} t$ and $\left(N^{n}, t\right) \models \neg \psi$. There are only two such points and they are the roots of the models $N^{n-1}$. On the other hand, we must have at least one of the following $\left(M_{a k}^{n}, r\right) \models[a] \psi$ or $\left(M_{a k}^{n}, r\right) \models[b] \psi$. This however is impossible, since in the first case there is a $z \in M_{a k}^{n}$ such that $r R_{a} z$ and $z$ is the root of the tree $N^{n-1}$; whereas, in the second case, there is a $u$ such that $r R_{b} u$ and $u$ is the root of the model $N^{n-1}$. In either case, the root of $T_{[\mathrm{V} \Gamma] \psi}^{\left.\left\langle\left(M_{a k}^{n}, r\right)\right\} \circ\left\{\left(N^{n}, r\right)\right\}\right\rangle}$ must have a child of the form $T_{\psi}^{\left\langle\mathbb{S} \cup\left\{\left(N^{n-1}, r\right)\right\} \circ\left\{\left(N^{n-1}, r\right)\right\} \cup \mathbb{T}\right\rangle}$ which is impossible since, using Proposition 1, we have two bisimilar models, namely $\left(N^{n-1}, r\right)$ for which $\left(N^{n-1}, r\right) \mid=\psi$ and $\left(N^{n-1}, r\right) \mid=\neg \psi$. The assumption that there is $[\vee \Gamma] \psi$ such that $\left(N^{n}, r\right) \models[\vee \Gamma] \psi$ and $\left(M_{w}^{n}, r\right) \models \neg[\vee \Gamma] \psi$ leads to a contradiction in a similar way.

(c) The assumption that there is such a formula plus the rule for formulas of the form $[\wedge \Gamma] \psi$ from Definition 6, leads to a contradiction as in item(b) above.

(d) Let $\phi, \mathbb{S}$, and $\mathbb{T}$ be as described and let $T_{\phi}^{\left\langle\mathbb{S} \cup\left\{\left(M_{w}^{n}, r\right)\right\},\left\{\left(N^{n}, r\right)\right\} \cup \mathbb{T}\right\rangle}$ be the extended syntax tree. Suppose $n>1^{4}$. We consider only the case $w=a k$. The case $w=b k$ is similar. Let $r t$ denote the root of the tree. It is obvious that the syntax label of $r t$ cannot be one of the following "T", " $\perp$ ", " $p$ ", and item (b) implies that $\operatorname{synl}(r t)$

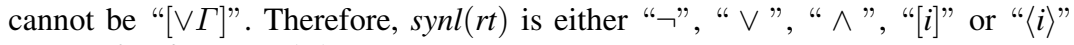
for $i \in\{a, b\}$. If $\operatorname{synl}(r t)$ is one of the Boolean connectives, then $r t$ will have as a child the root of a tree $T_{\psi}^{\left\langle\mathbb{S}_{1} \cup\left\{\left(M_{a k}^{n}, r\right)\right\},\left\{\left(N^{n}, r\right)\right\} \cup \mathbb{T}_{1}\right\rangle}$ or $T_{\psi}^{\left\langle\mathbb{T}_{1} \cup\left\{\left(N^{n}, r\right)\right\},\left\{\left(M_{a k}^{n}, r\right)\right\} \cup \mathbb{S}_{1}\right\rangle}$ for some sub-formula $\psi$ of $\phi$. The reasoning above shows that the root node $r t_{1}$ of this tree cannot have a syntactic label of the form "T", " $\perp$ ", " $p$ ", or "[V $\Gamma]$ ". Hence, $T_{\phi}^{\left\langle\mathbb{S} \cup\left\{\left(M_{a k}^{n}, r\right)\right\},\left\{\left(N^{n}, r\right)\right\} \cup \mathbb{T}\right\rangle}$ must contain at least one node that is the root of a tree $T_{\psi}^{\left\langle\mathbb{S}_{l} \cup\left\{\left(M_{a k}^{n}, r\right)\right\},\left\{\left(N^{n}, r\right)\right\} \cup \mathbb{T}_{l}\right\rangle}$ or $T_{\psi}^{\left\langle\mathbb{T}_{l} \cup\left\{\left(N^{n}, r\right)\right\},\left\{\left(M_{a k}^{n}, r\right)\right\} \cup \mathbb{S}_{l}\right\rangle}$ for some sub-formula $\psi$ of $\phi$, that has one of the forms $[a] \theta,\langle a\rangle \theta,\langle b\rangle \theta$ or $[b] \theta$. Let $n$ be the first such node in the enumeration of $T_{\phi}^{\left\langle\mathscr{S} \cup\left\{\left(M_{a k}^{n}, r\right)\right\},\left\{\left(N^{n}, r\right)\right\} \cup \mathbb{T}\right\rangle}$. It is easy to see that for all formulas $\langle b\rangle \theta$ and $[b] \theta,\left(M_{a k}^{n}, r\right) \mid=\langle b\rangle \theta$ if and only if $\left(N^{n}, r\right) \models\langle b\rangle \theta$, and $\left(M_{a k}^{n}, r\right) \models[b] \theta$ if and only if $\left(N^{n}, r\right) \mid=[b] \theta$. Hence, $\psi$ is either of the form $[a] \theta$ or $\langle a\rangle \theta$. Consider the tree $T_{\psi}^{\left\langle\mathbb{S}_{l} \cup\left\{\left(M_{a k}^{n}, r\right)\right\},\left\{\left(N^{n}, r\right)\right\} \cup \mathbb{T}_{l}\right\rangle}$. In this case, $\psi$ is not of the form $[a] \theta$ since the root of the tree would contain a child with semantic label $\mathbb{S}_{l} \cup\left\{\left(M_{a k}^{n}, N^{n-1}\right)\right\},\left\{\left(N^{n}, N^{n-1}\right)\right\} \cup$ $\left.\mathbb{T}_{l}\right\rangle$. Given the fact that $\left(M_{a k}^{n}, N^{n-1}\right)$ and $\left(N^{n}, N^{n-1}\right)$ are bisimilar, we arrive at a contradiction using Proposition 1 . Hence $\psi$ has the form $\langle a\rangle \theta$; moreover, the bisimilarity of $\left(M_{a k}^{n}, N^{n-1}\right)$ and $\left(N^{n}, N^{n-1}\right)$ implies that the root of the tree can only have a child with semantic label $\left\langle\mathbb{S}_{l+1} \cup\left\{\left(M_{a k}^{n}, M_{k}^{n-1}\right)\right\},\left\{\left(N^{n}, N^{n-1}\right)\right\} \cup \mathbb{T}_{l+1}\right\rangle$. It is obvious that this reasoning can be repeated until the branch $B$ such that $I(B)=a v$ is constructed. In the case of $T_{\psi}^{\left\langle\mathbb{T}_{l} \cup\left\{\left(N^{n}, r\right)\right\},\left\{\left(M_{a k}^{n}, r\right)\right\} \cup \mathbb{S}_{l}\right\rangle}$, similar considerations show that $\psi$ has the form $[a] \theta$ and that there is a branch $B$ such that $I(B)=a k$.

(e) The proof is analogous to the proof of item (d).

\footnotetext{
${ }^{4}$ The proof of the case $n=1$ will become clear from the proof for $n>1$
} 
Theorem 1. Let $\mathbb{M}$ denote the union of all $\mathbb{M}_{n}$ and $\mathbb{N}_{n}$ models. Let $\mathbb{O}$ denote the union of all $\mathbb{D}_{n}$ and $\mathbb{P}_{n}$ models. Then

(1) $[\wedge \Gamma] M L_{n}^{m} \Varangle_{\mathrm{M}}^{S U B X P}[\vee \Gamma] M L_{n}^{m}$.

(2) $[\vee \Gamma] M L_{n}^{m} \underset{\mathbb{O}}{S U B E X P}[\wedge \Gamma] M L_{n}^{m}$.

Proof. We prove (2). Item (e) from Lemma 1 implies that the extended syntax tree $T_{\delta}^{\left\langle\mathbb{D}^{\mathrm{m}}, \mathbb{P}^{\mathrm{n}}\right\rangle}$ of any $[\wedge \Gamma] M L_{n}^{m}$ formula $\delta$ such that $\mathbb{D}^{\mathrm{n}} \models \delta$ and $\mathbb{P}^{\mathrm{n}} \models \neg \delta$ contains $2^{n}$ different branches. Hence, any $[\wedge \Gamma] M L_{n}^{m}$ formula $\delta$ such that $\mathbb{D}=[\vee \Gamma]^{n} p \leftrightarrow \delta$ has size at least $2^{n}$. Since $[\vee \Gamma]^{n} p$ is equivalent to the formula $\chi_{n}$ from Definition 10 , we see that $[\vee \Gamma] M L_{n}^{m}$ is exactly exponentially more succinct than $[\wedge \Gamma] M L_{n}^{m}$.

The proof of (1) is analogous.

\section{Conclusion and Open Questions}

As mentioned in the introduction, the results presented here are relevant to epistemic logic. One disadvantage of such an interpretation is that all relations in our models are neither reflexive, nor symmetric, nor transitive, whereas the semantics of epistemic logic is usually given via models in which all relations are relations of equivalence. We believe that our results can be extended to this class of models as well, although we conjecture that the proofs will be technically more challenging than ours.

On a more general note. The study of representational succinctness is a vastly underdeveloped subject and many of the known results rely on unproven computational complexity conjectures (see for example [4]). Improving the proofs of these results so that they are not dependent on such conjectures will greatly increase our understanding of this phenomenon.

\section{References}

1. Adler, M., Immerman, N.: An $\mathrm{n}$ ! lower bound on formula size. ACM Transactions on Computational Logic, (2003) vol 4(3):296-314.

2. Blackburn, P., de Rijke, M., Venema, Y. Modal logic. Cambridge University Press, (2001).

3. French, T., van der Hoek, W., Iliev, P., Kooi, B. Succinctness of epistemic languages. In Proceedings of the 22nd International Joint Conference on Artificial Intelligence (IJCAI), (2011): 881-886.

4. Gogic, G., Papadimitriou, C., Selman, B., Kautz, H. The comparative linguistics of knowledge representation. In Proceedings of the 14th International Joint Conference on Artificial Intelligence (IJCAI), (1995): 862-869.

5. Grohe, M., Schweikardt, N.: The succinctness of first-order logic on linear orders. In Proceedings of the 19th Annual IEEE Symposium on Logic in Computer Science (LICS), (2004) : 438-447.

6. Lutz, C.: Complexity and succinctness of public announcement logic. In Proceedings of the 5th International Joint Conference on Autonomous Agents and Multiagent Systems (AAMAS), (2006) : $137-143$. 